\title{
Asymptotic Pairwise Dispersion Corrections Can Describe Layered Materials Accurately
}

\author{
A. Otero-de-la-Roza, ${ }^{1, *}$ Luc M. LeBlanc, ${ }^{2, \dagger}$ and Erin R. Johnson ${ }^{2}{ }^{\ddagger}$ \\ ${ }^{1}$ Departamento de Química Física y Analítica, Facultad de Química, Universidad de Oviedo, 33006 Oviedo, Spain \\ ${ }^{2}$ Department of Chemistry, Dalhousie University, \\ 6274 Coburg Rd, Halifax, Nova Scotia, B3H 4R2, Canada
}

(Dated: February 27, 2020)

Reference values: The reference interlayer lattice parameters $(c)$ were obtained from experimental geometries ${ }^{1}$ back-corrected for zero-point vibrational effects. ${ }^{2}$ The reference exfoliation energies are the Random-Phase Approximation (RPA) values given by Björkman, with the exception of h-BN. ${ }^{2}$ For h-BN, the reference value was taken from MP2 calculations ${ }^{3}$ that were shown to agree well with $\operatorname{CCSD}(\mathrm{T})$ for small kpoint meshes and are hence expected to be of greater accuracy than the RPA value, which is thought to be underestimated. ${ }^{3,4}$ The reference exfoliation energy for h-BN was converted to units of $\mathrm{meV} / \AA^{2}$ using in-plane lattice constants of $2.51 \AA$. It is important to note that the RPA also has limitations regarding accuracy ${ }^{4,5}$ but, as argued by Björkman, it is the best available benchmark data obtainable at the moment for layered materials.

Additional method details: For each layered material, a series of single-point energy calculations were performed for a range of interlayer separations, both compressed and expanded relative to experiment, by varying the $c$ lattice parameter. The in-plane unit cell dimensions (lattice parameters $a$ and $b$ ) were held fixed at the reference experimental values. The optimum interlayer separations and minimum energies were then obtained by polynomial interpolation, as described previously by Bjorkman. The exfoliation energy was evaluated as the difference between the minimum energy and the value in the limit of large interlayer separation. The calculations used density and planewave cut-offs of 100 and 1000 Ry, respectively, a cold-smearing parameter of 0.01 Ry $\left(\mathrm{NbTe}_{2}\right)$ and $0.001 \mathrm{Ry}$ (graphite, $\mathrm{TaS}_{2}, \mathrm{TaSe}_{2}, \mathrm{VS}_{2}$, and $\mathrm{VSe}_{2}$ ), and a $12 \times 12 \times 4$ k-point mesh.

Interlayer non-additivity effects: While negligi- ble for graphite, the magnitude of the interlayer nonadditivity effects is higher for transition-metal dichalcogenides, increasing down the chalcogen group. To quantify the magnitude of interlayer non-additivity effects, the XDM calculations were repeated using multipole moments and polarizabilities fixed at their values for infinitely separated layers. This was found to increase the exfoliation energies for all materials except graphite and, overall, it results in a slight worsening of the predicted geometries, with the MAE increasing to $0.107 \AA$. While the energies are slightly improved, with the MAE decreasing to $2.9 \mathrm{meV} / \AA^{2}$, this is due to a substantial improvement in the exfoliation energy of $\mathrm{PdTe}_{2}$. With this one exception, the variation in results is small and well within the uncertainties of the reference RPA calculations.

* aoterodelaroza@gmail.com

† luc.leblanc@dal.ca

‡ erin.johnson@dal.ca

[1] M. Hellenbrandt, Crystallogr. Rev. 10, 17 (2004).

[2] T. Björkman, J. Chem. Phys. 141, 074708 (2014).

[3] F. Hummel, T. Gruber, and A. Grüneis, Eur. Phys. J. B 89, 235 (2016).

[4] J. Paier, X. Ren, P. Rinke, G. Scuseria, A. Grüneis, G. Kresse, and M. Scheffler, New J. Phys. 14, 043002 (2012).

[5] E. R. Johnson, in Non-covalent Interactions in Quantum Chemistry and Physics, edited by A. Otero-de-la-Roza and G. A. DiLabio (Elsevier, 2017) Chap. 5, pp. 169-194.

[6] S. A. Tawfik, T. Gould, C. Stampfl, and M. J. Ford, Phys. Rev. Mater. 2, 034005 (2018). 
FIG. 1: The 11 compounds forming Tawfik's layered-material benchmark set. ${ }^{6}$ The unit cell boundary is shown in red. For graphite, the upper layer is shown in grey and the lower layer in black. Pink: boron, purple: nitrogen, yellow: chalcogen, blue: transition metal.

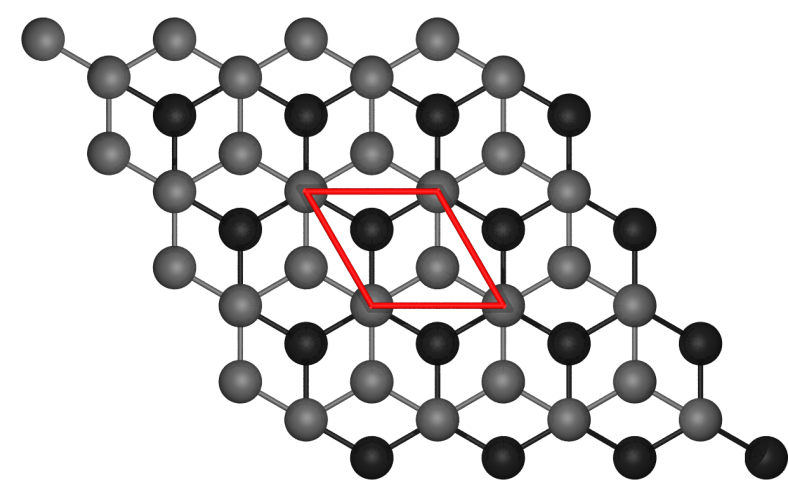

(a) Graphite

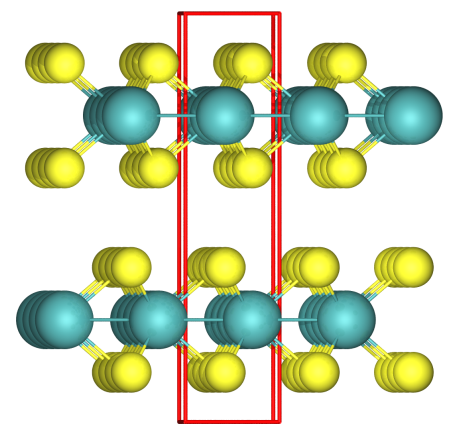

(c) $\operatorname{Mo}\{\mathrm{S}, \mathrm{Se}, \mathrm{Te}\}_{2}$ and $\mathrm{WS}_{2}$

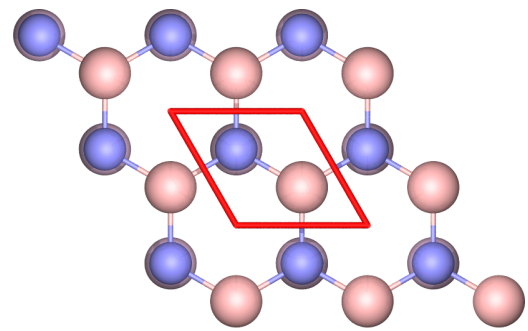

(b) h-BN

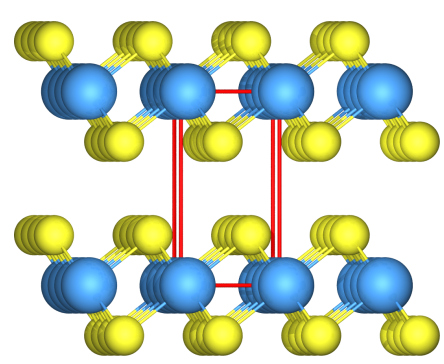

(d) $\mathrm{TaS}_{2}, \operatorname{Hf}\{\mathrm{S}, \mathrm{Se}, \mathrm{Te}\}_{2}$, and $\mathrm{PdTe}_{2}$ 
TABLE I: Mean absolute errors (MAE) and mean absolute percent errors (MAPE) in the inter-layer lattice parameters $(\AA)$ and exfoliation energies $\left(\mathrm{meV} / \AA^{2}\right)$ for the set of 11 layered materials studied by Tawfik et al. ${ }^{6}$

\begin{tabular}{|c|c|c|c|c|}
\hline \multirow[t]{2}{*}{ Method } & \multicolumn{2}{|c|}{ Geometries } & \multicolumn{2}{|c|}{ Energies } \\
\hline & MAE & MAPE & MAE & MAPE \\
\hline \multicolumn{5}{|c|}{ DFT-D functionals } \\
\hline${\mathrm{PBE}-\mathrm{D} 2^{a}}^{a}$ & 0.173 & 2.4 & 9.4 & 49.0 \\
\hline PBE-D3 $(0)^{a}$ & 0.139 & 1.8 & 7.5 & 37.3 \\
\hline PBE-D3(BJ) ${ }^{a}$ & 0.187 & 2.0 & 11.5 & 56.6 \\
\hline PBE-D3(BJ) $+\mathrm{ATM}^{a}$ & 0.130 & 1.5 & 6.0 & 29.6 \\
\hline \multicolumn{5}{|c|}{ XDM and XDM-like } \\
\hline $\mathrm{PBE} \mathrm{XDM}^{a}$ & 0.088 & 1.0 & 3.2 & 14.1 \\
\hline B86bPBE-XDM $^{a}$ & 0.072 & 0.9 & 4.0 & 18.2 \\
\hline PBE-dDsC ${ }^{b}$ & 0.291 & 3.3 & 1.8 & 9.2 \\
\hline \multicolumn{5}{|c|}{ TS and MBD-based } \\
\hline${\mathrm{PBE}-\mathrm{TS}^{b}}^{b}$ & 0.080 & 0.9 & 11.4 & 59.0 \\
\hline PBE-SCS-TS ${ }^{b}$ & 0.135 & 1.4 & 6.7 & 35.2 \\
\hline PBE-MBD-FIA $^{b}$ & 0.124 & 1.5 & 3.6 & 15.5 \\
\hline \multicolumn{5}{|c|}{ Non-local vdW functionals } \\
\hline $\mathrm{C} 09-\mathrm{DF} 1^{c}$ & 0.078 & 1.0 & 7.5 & 36.8 \\
\hline $\mathrm{cx} 13-\mathrm{DF} 1^{c}$ & 0.037 & 0.4 & 3.2 & 15.7 \\
\hline optB88-DF $2^{b}$ & 0.112 & 1.4 & 6.1 & 30.9 \\
\hline $\mathrm{vdW}-\mathrm{DF} 2^{b}$ & 0.698 & 8.3 & 2.9 & 11.2 \\
\hline optPBE-DF $2^{b}$ & 0.400 & 4.7 & 3.1 & 15.5 \\
\hline optPBE-DF $2^{c}$ & 0.366 & 4.2 & 2.3 & 10.3 \\
\hline optB88-DF2 ${ }^{c}$ & 0.177 & 2.0 & 3.7 & 19.6 \\
\hline optB86b-DF $2^{c}$ & 0.096 & 1.1 & 4.2 & 21.7 \\
\hline revB86b-DF $2^{c}$ & 0.081 & 0.9 & 1.8 & 9.9 \\
\hline SCAN-rVV $10^{b}$ & 0.075 & 0.9 & 1.5 & 7.4 \\
\hline
\end{tabular}

${ }^{a}$ This work; ${ }^{b}$ obtained from the work of Tawfik et al., the calculated values were kindly provided by the authors; ${ }^{c}$ obtained from the work of Björkman. ${ }^{2}$

TABLE II: Error statistics for the lattice parameters (in $\AA$ ) and exfoliation energies (in $\mathrm{meV} / \AA^{2}$ ) for the full set of 26 layered materials studied by Björkman. ${ }^{2}$

\begin{tabular}{lcrrr}
\hline \hline Method & \multicolumn{2}{c}{ Geometries } & \multicolumn{2}{c}{ Energies } \\
& MAE & MAPE & MAE & MAPE \\
\hline \hline \multicolumn{5}{c}{ DFT-D functionals } \\
\hline PBE-D2 $^{a}$ & 0.179 & 2.8 & 11.1 & 56.7 \\
PBE-D3(0) $^{a}$ & 0.136 & 2.2 & 9.2 & 46.3 \\
PBE-D3(BJ) $^{a}$ & 0.206 & 3.0 & 14.2 & 71.1 \\
${\text { PBE-D3(BJ) }+ \text { ATM }^{a}}_{5} 0.140$ & 2.5 & 7.8 & 39.4 \\
\hline \multicolumn{5}{c}{ XDM $^{5}$ and XDM-like } \\
\hline PBE-XDM $^{a}$ & 0.098 & 1.4 & 4.3 & 21.2 \\
B86bPBE-XDM $^{a}$ & 0.107 & 1.6 & 4.6 & 22.6 \\
\hline \multicolumn{5}{c}{ Non-local vdW functionals } \\
\hline C09-DF1 $^{b}$ & 0.096 & 1.5 & 8.6 & 43.5 \\
cx13-DF1 $^{b}$ & 0.056 & 0.8 & 4.1 & 20.8 \\
optPBE-DF2 $^{b}$ & 0.344 & 4.6 & 2.0 & 10.0 \\
optB88-DF2 $^{b}$ & 0.158 & 2.0 & 3.9 & 20.7 \\
optB86b-DF2 $^{b}$ & 0.080 & 1.0 & 4.8 & 24.9 \\
revB86b-DF2 $^{b}$ & 0.074 & 1.0 & 2.7 & 14.4 \\
\hline \hline
\end{tabular}

${ }^{a}$ This work; ${ }^{b}$ obtained from the work of Björkman. 
TABLE III: Calculated and reference inter-layer lattice parameters $(\AA)$ for the 26 systems in Björkman's test set. The subset of 11 systems studied by Tawfik are indicated in bold.

\begin{tabular}{|c|c|c|c|c|c|c|c|}
\hline System & $\overline{\text { Ref. }}$ & $\overline{\text { B86bPBE-XDM }}$ & $\overline{\overline{\text { PBE-XDM }}}$ & $\overline{\text { PBE-D2 }}$ & $\overline{\text { PBE-D3(0) }}$ & 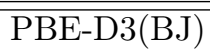 & PBE-D3(BJ)+ATM \\
\hline Graphite & 6.63 & 6.69 & 6.86 & 6.46 & 6.94 & 6.72 & 6.80 \\
\hline h-BN & 6.63 & 6.61 & 6.70 & 6.17 & 6.78 & 6.65 & 6.65 \\
\hline HfS2 & 5.83 & 5.75 & 5.84 & 5.72 & 5.93 & 5.77 & 5.80 \\
\hline HfSe2 & 6.15 & 6.03 & 6.05 & 5.96 & 6.13 & 6.03 & 6.03 \\
\hline HfTe2 & 6.64 & 6.72 & 6.74 & 6.65 & 6.76 & 6.59 & 6.65 \\
\hline MoS2 & 12.28 & 12.31 & 12.43 & 12.49 & 12.42 & 12.11 & 12.23 \\
\hline MoSe2 & 12.91 & 12.79 & 12.84 & 12.88 & 12.83 & 12.49 & 12.68 \\
\hline MoTe2 & 13.96 & 13.91 & 13.90 & 13.72 & 13.74 & 13.42 & 13.50 \\
\hline NbSe2 & 12.53 & 12.49 & 12.52 & 12.76 & 12.49 & 12.33 & 12.36 \\
\hline NbTe2 & 6.61 & 6.39 & 6.39 & 6.47 & 6.28 & 6.18 & 6.22 \\
\hline $\mathrm{PbO}$ & 4.99 & 5.15 & 4.97 & 5.09 & 5.26 & 4.97 & 5.15 \\
\hline PdTe2 & 5.11 & 5.15 & 5.10 & 4.88 & 4.88 & 4.89 & 4.91 \\
\hline PtS2 & 5.04 & 5.05 & 5.08 & 4.33 & 4.52 & 4.64 & 4.62 \\
\hline PtSe2 & 5.07 & 5.32 & 5.31 & 4.67 & 4.84 & 4.87 & 4.94 \\
\hline TaS2 & 5.89 & 5.88 & 5.87 & 5.79 & 5.88 & 5.76 & 5.78 \\
\hline TaSe2 & 6.27 & 6.07 & 6.11 & 6.02 & 6.10 & 5.91 & 5.99 \\
\hline TiS2 & 5.70 & 5.59 & 5.62 & 5.81 & 5.72 & 5.53 & 5.58 \\
\hline TiSe2 & 6.00 & 5.92 & 5.94 & 6.10 & 6.02 & 5.83 & 5.89 \\
\hline TiTe2 & 6.49 & 6.51 & 6.48 & 6.60 & 6.47 & 6.31 & 6.35 \\
\hline VS2 & 5.75 & 5.62 & 5.64 & 5.84 & 5.74 & 5.51 & 5.55 \\
\hline VSe2 & 6.10 & 6.04 & 6.07 & 6.23 & 6.16 & 5.89 & 5.93 \\
\hline WS2 & 12.31 & 12.48 & 12.47 & 12.16 & 12.47 & 12.08 & 12.26 \\
\hline WSe2 & 12.95 & 13.19 & 13.10 & 12.85 & 12.91 & 12.70 & 12.89 \\
\hline ZrS2 & 5.80 & 5.69 & 5.73 & 5.90 & 5.86 & 5.70 & 5.74 \\
\hline ZrSe2 & 6.12 & 5.96 & 6.00 & 6.11 & 6.12 & 5.92 & 5.97 \\
\hline $\mathrm{ZrTe} 2$ & 6.66 & 6.44 & 6.41 & 6.47 & 6.43 & 6.28 & 6.32 \\
\hline ME & & 0.0258 & 0.0099 & 0.0887 & 0.0293 & 0.2057 & 0.1404 \\
\hline MAE & & 0.1073 & 0.0976 & 0.1790 & 0.1365 & 0.2138 & 0.1670 \\
\hline $\operatorname{MRE}(\%)$ & & -0.43 & -0.24 & -1.52 & -0.52 & -2.84 & -2.01 \\
\hline MARE(\%) & & 1.59 & 1.40 & 2.77 & 2.12 & 2.96 & 2.47 \\
\hline
\end{tabular}


TABLE IV: Calculated and reference exfoliation energies $\left(\mathrm{meV} / \AA^{2}\right)$ for the 26 systems in Björkman's test set. The subset of 11 systems studied by Tawfik are indicated in bold.

\begin{tabular}{|c|c|c|c|c|c|c|c|}
\hline System & Ref. & B86bPBE-XDM & PBE-XDM & PBE-D2 & PBE-D3(0) & PBE-D3(BJ) & PBE-D3(BJ)+ATM \\
\hline Graphite & 18.32 & 20.57 & 18.57 & 21.09 & 18.36 & 20.26 & 16.37 \\
\hline h-BN & 21.60 & 21.32 & 20.19 & 28.06 & 17.89 & 19.07 & 15.67 \\
\hline HfS2 & 16.13 & 21.46 & 20.22 & 26.99 & 20.92 & 23.84 & 19.67 \\
\hline HfSe2 & 17.09 & 21.47 & 20.79 & 31.56 & 23.43 & 26.48 & 21.99 \\
\hline HfTe2 & 18.68 & 20.32 & 21.69 & 34.96 & 25.17 & 29.95 & 25.51 \\
\hline MoS2 & 20.53 & 23.54 & 20.83 & 18.05 & 28.32 & 32.43 & 23.65 \\
\hline MoSe2 & 19.63 & 22.99 & 21.03 & 24.96 & 31.43 & 35.58 & 26.35 \\
\hline MoTe2 & 20.80 & 19.82 & 19.56 & 32.29 & 31.17 & 37.89 & 29.56 \\
\hline NbSe2 & 19.57 & 32.11 & 31.25 & 29.83 & 38.14 & 43.28 & 35.13 \\
\hline $\mathrm{NbTe} 2$ & 23.03 & 27.42 & 28.48 & 36.31 & 39.43 & 47.68 & 38.90 \\
\hline $\mathrm{PbO}$ & 20.25 & 22.40 & 27.04 & 42.43 & 20.66 & 25.57 & 22.67 \\
\hline PdTe2 & 40.17 & 25.07 & 27.15 & 46.12 & 48.97 & 55.36 & 47.24 \\
\hline PtS2 & 20.55 & 18.71 & 17.14 & 52.68 & 36.83 & 38.68 & 28.42 \\
\hline PtSe2 & 19.05 & 19.27 & 18.72 & 51.66 & 37.27 & 39.57 & 30.53 \\
\hline TaS2 & 17.68 & 24.89 & 22.90 & 31.81 & 29.97 & 35.47 & 28.16 \\
\hline TaSe2 & 19.44 & 24.64 & 23.54 & 37.37 & 32.52 & 37.88 & 30.35 \\
\hline TiS2 & 18.88 & 27.71 & 25.47 & 14.24 & 25.72 & 32.39 & 25.94 \\
\hline TiSe2 & 17.39 & 26.62 & 25.23 & 19.84 & 27.32 & 34.01 & 27.73 \\
\hline TiTe2 & 19.76 & 24.24 & 25.15 & 27.99 & 28.88 & 38.44 & 32.54 \\
\hline VS2 & 25.61 & 28.69 & 27.88 & 18.27 & 30.38 & 38.82 & 30.74 \\
\hline VSe2 & 22.26 & 26.31 & 26.31 & 23.81 & 31.40 & 39.26 & 31.40 \\
\hline WS2 & 20.24 & 20.53 & 19.14 & 32.91 & 30.15 & 35.05 & 26.06 \\
\hline WSe2 & 19.98 & 20.27 & 19.63 & 37.80 & 33.50 & 36.95 & 27.50 \\
\hline ZrS2 & 16.98 & 23.08 & 22.20 & 12.66 & 21.04 & 25.02 & 20.53 \\
\hline ZrSe2 & 18.53 & 23.42 & 22.94 & 18.27 & 23.90 & 28.13 & 23.25 \\
\hline ZrTe2 & 16.34 & 23.60 & 24.71 & 27.26 & 27.25 & 34.62 & 29.51 \\
\hline $\mathrm{ME}$ & & -3.15 & -2.66 & -9.64 & -8.90 & -13.97 & -7.19 \\
\hline MAE & & 4.55 & 4.27 & 11.11 & 9.19 & 14.16 & 7.80 \\
\hline $\operatorname{MRE}(\%)$ & & 18.61 & 15.78 & 49.61 & 44.98 & 70.22 & 36.49 \\
\hline MARE(\%) & & 22.65 & 21.19 & 56.70 & 46.30 & 71.12 & 39.42 \\
\hline
\end{tabular}

